12. Kakimoto $\mathrm{K}$, Inoue $\mathrm{T}$, Nishikawa $\mathrm{T}$, Ishida $\mathrm{K}$, Kawakami K, Kuramoto T, et al. Primary CD56+ NK/T-cell lymphoma of the rectum accompanied with refractory ulcerative colitis. $J$ Gastroenterol. 2008;43:576-80.

13. Loehr WJ, Mujahed Z, Zahn FD, Gray GF, Thorbjarnarson B. Primary lymphoma of the gastrointestinal tract: a review of 100 cases. Ann Surg. 1969;170:232-8.

14. Friedman HB, Silver GM, Brown CH. Lymphoma of the colon simulating ulcerative colitis. Report of four cases. Am J Dig Dis. 1968;13:910-7.

15. Dawson IM, Cornes JS, Morson BC. Primary malignant lymphoid tumours of the intestinal tract. Report of 37 cases with a study of factors influencing prognosis. Br J Surg. 1961;49:80-9.

16. Grünberger B, Wöhrer S, Streubel B, Formanek M, Petkov $\mathrm{V}$, Puespoek A, et al. Antibiotic treatment is not effective in patients infected with Helicobacter pylori suffering from extragastric MALT lymphoma. J Clin Oncol. 2006;24:1370-5.

17. Guney N, Basaran M, Aksakalli N, Bavbek S, Erseven G. Primary non-Hodgkin's lymphoma of the rectum. Onkologie. 2007;30:385-7.

\section{Volvulus of the transverse colon}

\section{Introduction}

Colonic volvulus accounts for less than $5 \%$ of all cases of intestinal obstruction. ${ }^{1}$ It usually occurs in the sigmoid colon and transverse colon volvulus (TCV) is probably the rarest form of colonic volvulus, accounting for less than $11 \%$ of all cases of colonic volvulus, but with the highest mortality. ${ }^{1,2}$

\section{Case report}

A 38-years-old male patient presented to the emergency with sudden onset upper abdominal pain, bilious vomiting and obstipation for duration of one day. There was no previous history of similar complaints, although the patient did give history of chronic constipation and substance abuse (proxyvon tablets) for the last eight years.

Plain X-ray of the abdomen revealed a twisted loop of colon lying in the upper midline (Figure 1). He was taken up for laparotomy with a diagnosis of large bowel obstruction due to volvulus, possibly of the transverse colon. At laparotomy, a grossly dilated and gangrenous transverse colon was found, which was twisted around itself in a clockwise turn (Figure 2). The gangrenous colon was resected and its proximal end brought out as a colostomy. The patient had an uneventful recovery and was discharged in a satisfactory condition.

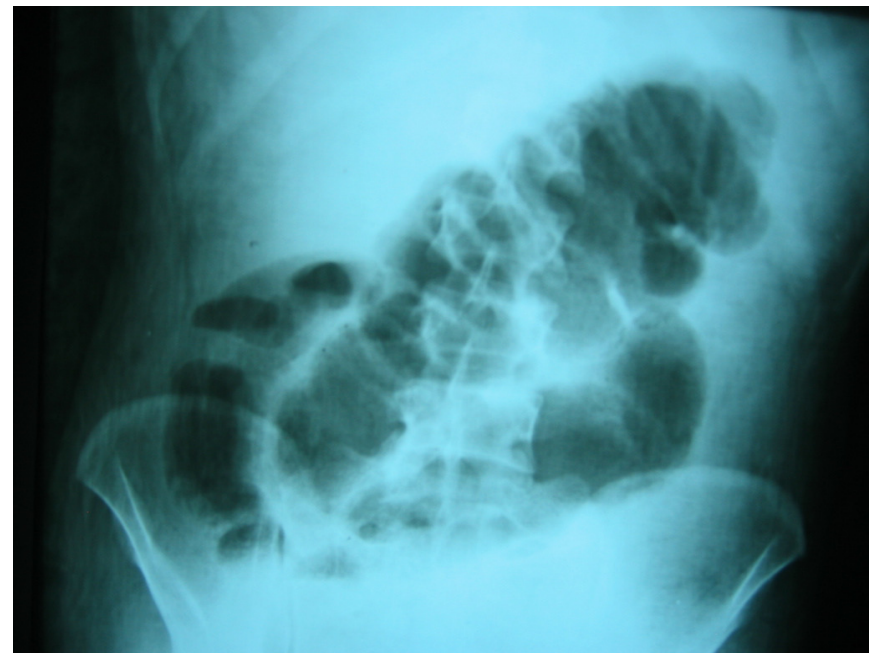

Figure 1: Plain X-ray of the abdomen revealing a twisted loop of colon lying in the upper midline

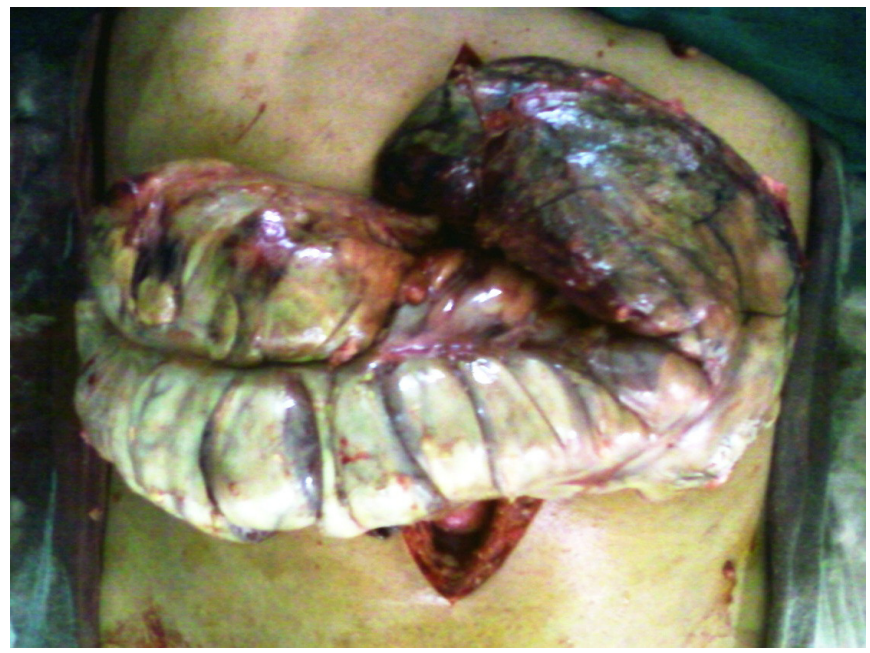

Figure 2: Grossly dilated and gangrenous transverse colon twisted around itself in a clockwise turn as seen during laparotomy

\section{Discussion}

Transverse colon volvulus (TCV) was first described by Kallio in $1932^{2}$ and still remains a very uncommon cause of intestinal obstruction. ${ }^{2}$ Classically, TCV is said to occur twice as much commonly in females than in males; in the second and third decades of life. Some authors have reported an additional peak in the seventh decade of life.,

In a normal situation, the transverse colon has a short mesocolon and is fixed at both its ends (the hepatic and splenic flexures), thus being less prone to undergoing volvulus. However, in the presence of various factors, such as congenital (abnormal fixity of the mesentery, congenital errors of midgut rotation); mechanical (previous surgery, adhesions, distal obstruction) and physiological (chronic constipation, 
pregnancy, colitis), it can rotate, leading to a closed loop obstruction, gangrene or even perforation in neglected cases. $^{2-5}$

The diagnosis is rarely made preoperatively. Plain abdominal X-rays may show massive dilatation of the proximal colon with an empty distal bowel and two air-fluid levels caused by double closed-loop obstruction (at the level of the transverse colon and cecum), or a 'bent inner tube appearance'; however, plain X-rays are not very sensitive, and may not contribute to the diagnosis. In such cases, a barium enema study may help in the diagnosis by showing the typical 'bird's beak' appearance. ${ }^{4,6}$ Computerised tomogram scan (CT scan) has the highest sensitivity, and will help delineate the closed loop obstruction, marked dilatation of the proximal colon and collapse of the distal portion of the transverse/descending colon, as well as the twisting of the mesenteric vessels, all of which suggest a TCV. ${ }^{3,7}$ Although there are occasional reports of successful conservative management ${ }^{8}$ of $\mathrm{TCV}$, surgery is usually required in the vast majority of cases. Depending on the status of the colon, the surgical options may vary from simple derotation (and colopexy) in patients with a viable colon, to resection (with or without primary anastomosis) in the presence of gangrene or perforation. Resection of the redundant transverse colon has the least chance of recurrence.

\section{ROBIN KAUSHIK MAYANK JAYANT \\ Correspondence: Dr. Robin Kaushik Department of Surgery Government Medical College and Hospital, Chandigarh, India \\ Email: robinkaushik@yahoo.com}

\section{References}

1. Booij KAC, Tanis PJ, van Gulik TM, Gouma DJ. Recurrent volvulus of the transverse colon after sigmoid resection. Int $J$ Colorectal Dis. 2009; 24:471-2.

2. Newton NA, Reines HD. Transverse colon volvulus: case reports and review. AJR Am J Roentgenol. 1977;128:69-72.

3. Younes N, Al-Ardah MI. Transverse colon volvulus: acse report and review of literature. Pak J Med Sci. 2010;26:716-9.

4. Ciraldo A, Thomas D, \& Schmidt S. A case report: transverse colon volvulus associated with chilaiditis syndrome. The Internet Journal of Gastroenterol. 2000:1.

5. Houshian S, Sorensen JS, Jensen KE. Volvulus of the transverse colon in children. J Pediatr Surg. 1998;33:1399-140.

6. Mortensen NJ, Hoffman G. Volvulus of the transverse colon. Postgrad Med J. 1979;55:54-7.
7. Matsushima K, Suzuki Y. Transverse colon volvulus and associated Chilaiditi's syndrome. Am J Surg. 2006;192:203-4.

\section{Caecal volvulus: a cause for intestinal obstruction}

\section{Introduction}

Caecal volvulus is a rare clinical condition causing intestinal obstruction. It is responsible for $1 \%$ of all adult intestinal obstructions and $30 \%$ of all cases of volvulus involving the colon. The most common signs associated with it are abdominal pain, constipation and vomiting; none of which are specific for the condition. ${ }^{1}$ Abdominal radiography permits diagnosis in $70 \%$ of cases by 3 typical signs: caecum dilatation, a single air-fluid level in the right lower quadrant, and absence of gas in the colon. ${ }^{2}$ The CT scan typically shows a massively dilated caecum with associated small bowel dilation. The twisted or "whirl" mesenteric configuration around the ileocolic artery is pathognomonic of axial caecal volvulus. Overall, radiographic studies confirm the diagnosis of caecal volvulus $90 \%$ of the time. The remainder are diagnosed at surgery. ${ }^{3}$ In this report we present the case of a 70-year-old woman with caecal volvulus.

\section{Case report}

A 70-year-old woman presented with acute pain abdomen, vomiting and constipation since one day. The pain was diffuse and colicky in nature. She had no co-morbid illness and no history of surgery in the past.

$\mathrm{X}$-ray abdomen (erect and supine) (Figure 1) showed a loop of dilated large bowel on the right side with multiple loops of dilated small bowel showing air fluid levels. CT abdomen and pelvis (Figure 2) revealed a large caecal volvulus with an obstructed proximal bowel and a decompressed distal colon with malrotation of the gut.

An emergency laparotomy was performed which confirmed the presence of caecal volvulus and malrotation of the gut. The caecum was massively dilated with a few gangrenous patches (Figure 3). Detorsion and a right hemicolectomy with primary anastomosis using a stapler was performed.

The post-operative recovery was uneventful and the patient was discharged after 8 days. 